\title{
Parenteral Omega-3 Fatty Acid Supplementation Improves Outcome of Sepsis: A Real-World, Retrospective Cohort Study
}

\section{Youlian Chen (ロ 381904698@qq.com )}

Shenzhen People's Hospital

HuaiSheng Chen

Shenzhen People's Hospital

YiChun Jiang

Shenzhen People's Hospital

XueMing Tang

Shenzhen People's Hospital

YongHui Ma

Shenzhen People's Hospital

Xueyan Liu

Shenzhen People's Hospital

Wei Wang

Shenzhen People's Hospital

Research

Keywords: Sepsis, Omega-3 fatty acid, death, survival.

Posted Date: May 19th, 2021

DOI: https://doi.org/10.21203/rs.3.rs-487025/v1

License: (c) (i) This work is licensed under a Creative Commons Attribution 4.0 International License. Read Full License 


\section{Abstract}

Objectives: The role of omega-3 fatty acids in the treatment of sepsis is always on paradox. we tried to retrieve and download the patients' data in a certain period through the hospital information system, used data sorting so as to screen out the patients with sepsis so as to find out the role of omega- 3 fatty acids in sepsis.

Methods: Through the hospital information system, retrieve and include the patients who were admitted to the Department of critical medicine of Shenzhen People's Hospital from December 2016 to June 2019, screen out patients diagnosed with sepsis according to a certain criterion. The patients were grouped by whether they were applicated with omega-3 fatty acid or not.

Results: A total of 1733 cases included into analysis, among of whom 303 cases were applicated with omega-3 fatty acid. The amounts and baseline conditions between both groups were imbalance. Severity of omega-3 fatty acid group was higher than that of control group. Chi-square test found that the mortality rate of omega-3 fatty acid was higher than that of control group $(p<0.0001)$. But age, gender, whether there is abdominal infection, whether there is septicemia, shock, the need for mechanical ventilation, and the need for renal replacement therapy may all affect the prognosis of the patients. If these factors were used as covariates, multiple logistic regression analysis showed that there was no significant difference in mortality rate between the treatment group and the control group $(P=0.574)$. Survival analysis showed that the survival rate of treatment group was higher than that of the control group when at the end of total treatment duration $(P=0.035)$.

Conclusion: For patients with more severe sepsis, doctors are more likely to use omega- 3 fatty acids in the early stage. Omega-3 fatty acids may improve the long-term prognosis of sepsis, but the conclusion still needs to be accepted carefully.

\section{Background}

The therapeutic effect of Omega-3 fatty acid on sepsis and its mechanism are still unclear. Sepsis is a type of systemic inflammatory response cause to serious infection, that might lead to death by multiple organ failure[1]. Animal and in vitro studies showed Omega-3 fatty acid has a certain anti-inflammatory effect, thus, Omega-3 fatty acid has been recommended for the treatment of sepsis. It has discovered that Omega-3 fatty acid had anti-inflammatory effect via regulation of chemotaxis, cell express adhesion, leukocytes and endothelial cell adhesion, more than that it can change cell membrane phospholipids, inhibit expression of inflammatory genes and active peroxidase[2]. However there is a discrepancy between the results obtained in experimental and clinical studies. Former meta-analysis and systematic reviews had evaluated the role of Omega-3 on sepsis. Mo et al included a total of 721 patients in 12 randomized controlled trail, the analysis results indicated parenteral Omega-3 fatty acid supplementation can decreased the mortality of patients with sepsis, as well as shorten the hospital stay[3]. Our group evaluated two different series of sepsis patients with intestinal failure by randomized controlled trial, the 
results indicated that the efficacy of Omega-3 on sepsis are unpredictable[4, 5]. Especially in the second trail, Omega-3 didn't prevent the 28 days morality, however the treatment group had lower death rate in 60 days. The treatment group, which had lower CD3 T lymphocytes cells at the beginning of treatment, had equal level of lymphocytes with control after 7 days[5]. Aimed to explore weather Omega-3 fatty acid can promote lymphocytes cells recovery on sepsis and improve long-term prognosis, subsequently another mata-analyses had been performed. In this analyses more than double randomized controlled trial over Mo's had include. Sepsis and sepsis-induced acute respiratory distress syndrome(ARDS) was assess, the result suggested no matter transintestinal or parenteral Omega-3 fatty acid supplement, the sepsis morality had not improved[6].

With the advent of the big data era,a database was established in our hospital. In this study patient data for a certain period of time was download. Sepsis patients were screened and grouped according to whether they use parenteral supplementation of Omega-3 fatty acids. Through study and analyze these cases we try to find the effect of omega-3 fatty acids on sepsis.

\section{Materials And Methods}

Inclusion criteria

Patient's data who were admitted to the Intensive Medicine Department of Shenzhen People's Hospital from December 2016 to June 2019 were collected according to the following clinical features(Table 1). Non-sepsis patients were excluded. Each patient was assessed as to whether it clearly met the ICD-9 criterion. The research was carried out with the consent of the ethics committee of our hospital, and each patient had signed informed consent form agreeing to accept medical treatment, teaching, scientific research and other obligations. 
Table 1

Screening criteria for patients with sepsis

\begin{tabular}{|c|c|}
\hline evaluation indicator & Explanation \\
\hline infected focus * & With traceable infection site \\
\hline Abnormal leukocyte count ** & Leukocyte count $>12 \times 10^{9} / \mathrm{L}$ or $<4 \times 10^{9} / \mathrm{L}$ \\
\hline Abnormal respiratory rate ${ }^{\star \star}$ & Respiratory rate $>20$ beat per min,or need mechanical ventilation. \\
\hline Shock*** & MAP $<60 \mathrm{mmHg}$, or norepinephrine is needed. \\
\hline Abnormal renal function** & $\begin{array}{l}\mathrm{SCr}>144 \mathrm{umol} / \mathrm{L} \text {,or continuous renal replacement treatment is } \\
\text { needed. }\end{array}$ \\
\hline Coagulation disorders ** & Platelet count $<100 \times 10^{9} / \mathrm{L}$ \\
\hline Abnormal liver function** & TBIL > 34.1umol/l. \\
\hline 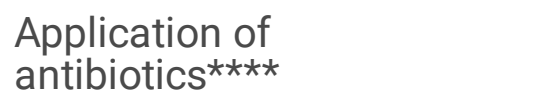 & Carbapenems or $\beta$ - lactams are preferred. \\
\hline
\end{tabular}


Table 2

Patients' baseline comparison

\begin{tabular}{|c|c|c|c|c|c|}
\hline & $\mathbf{N}$ & $\begin{array}{l}\text { Omega-3 fatty acid } \\
\text { group }\end{array}$ & $\begin{array}{l}\text { Control } \\
\text { group }\end{array}$ & $\begin{array}{l}\text { Statistical } \\
\text { value ** }\end{array}$ & $\begin{array}{l}P \\
\text { value }\end{array}$ \\
\hline Gender(female) & 720 & 100 & 620 & $x^{2}=11.04$ & 0.0001 \\
\hline Age & 1733 & $67 \pm 19$ & $61 \pm 20$ & $t=7.029$ & 0.0001 \\
\hline \multicolumn{6}{|l|}{ Infectious foci } \\
\hline Lung infection & 1098 & 192 & 906 & $x^{2}=0.0001$ & 1.000 \\
\hline Abdominal infection & 83 & 34 & 49 & $x^{2}=33.312$ & 0.0001 \\
\hline Biliary tract infection & 47 & 10 & 37 & $x^{2}=0.482$ & 0.442 \\
\hline Urinary tract infection & 8 & 1 & 7 & $X^{2}=0.138$ & 1.000 \\
\hline Septicemia & 220 & 70 & 150 & $x^{2}=35.887$ & 0.001 \\
\hline Other infectious sites & 765 & 135 & 630 & $x^{2}=0.025$ & 0.899 \\
\hline Mechanical ventilation & 1067 & $221(72.94)$ & $846(59.16)$ & $x^{2}=20.055$ & 0.0001 \\
\hline Shock & 1458 & $278(91.75)$ & $1190(83.22)$ & $X^{2}=14.052$ & 0.0001 \\
\hline $\begin{array}{l}\text { Renal replacement } \\
\text { treatment }\end{array}$ & 345 & $96(31.68)$ & $249(17.41)$ & $x^{2}=31.934$ & 0.0001 \\
\hline \multicolumn{6}{|l|}{$\begin{array}{l}\text { Laboratory } \\
\text { examination* }\end{array}$} \\
\hline WBC $\left(\times 10^{9} / \mathrm{L}\right)$ & 1479 & $12.94 \pm 8.95$ & $13.20 \pm 8.47$ & $Z=-1.285$ & 0.199 \\
\hline $\mathrm{N}(\%)$ & 1470 & $86.16 \pm 10.18$ & $\begin{array}{l}83.62 \pm \\
11.99\end{array}$ & $Z=-3.735$ & 0.0001 \\
\hline $\mathrm{L}(\%)$ & 1470 & $8.92 \pm 8.07$ & $10.96 \pm 9.98$ & $Z=-3.964$ & 0.0001 \\
\hline$M(\%)$ & 1470 & $8.92 \pm 8.07$ & $10.96 \pm 9.98$ & $Z=-3.964$ & 0.0001 \\
\hline $\mathrm{hsCRP}(\mathrm{mg} / \mathrm{L})$ & 114 & $103.94 \pm 86.31$ & $\begin{array}{l}70.53 \pm \\
85.64\end{array}$ & $Z=-2.242$ & 0.025 \\
\hline
\end{tabular}

Note: * WBCIwhite blood cell. N: percentage of neutrophil. L: percentage of lymphocyte. M: percentage of monocyte. hsCRP: high sensitive $\mathrm{C}$ reactive protein.

PCT: procalcitonin. BUN: blood urea nitrogen. Cr: creatinine. TBIL: total bilirubin. PLT: platelet. AT III: antithrombin III.

** $X^{2}$ : Chi-square. t: t-test. Z: Mann Whitney test. 


\begin{tabular}{|c|c|c|c|c|c|}
\hline & $\mathbf{N}$ & $\begin{array}{l}\text { Omega-3 fatty acid } \\
\text { group }\end{array}$ & $\begin{array}{l}\text { Control } \\
\text { group }\end{array}$ & $\begin{array}{l}\text { Statistical } \\
\text { value **}\end{array}$ & $\begin{array}{l}P \\
\text { value }\end{array}$ \\
\hline PCT(ng/ml) & 494 & $14.71 \pm 32.78$ & $\begin{array}{l}11.51 \pm \\
28.97\end{array}$ & $Z=-2.655$ & 0.008 \\
\hline $\mathrm{BUN}(\mathrm{mmol} / \mathrm{L})$ & 1405 & $11.96 \pm 10.62$ & $8.87 \pm 8.00$ & $t=5.185$ & 0.0001 \\
\hline $\mathrm{Cr}(\mathrm{umol} / \mathrm{L})$ & 1405 & $172.13 \pm 200.23$ & $\begin{array}{l}150.16 \pm \\
192.55\end{array}$ & $Z=-1.666$ & 0.096 \\
\hline TBIL(umol/L) & 222 & $25.19 \pm 43.30$ & $\begin{array}{l}20.99 \pm \\
32.06\end{array}$ & $Z=-1.633$ & 0.102 \\
\hline $\operatorname{PLT}\left(\times 10^{9} / \mathrm{L}\right)$ & 1480 & $188.80 \pm 115.03$ & $\begin{array}{l}202.51 \pm \\
109.48\end{array}$ & $t=-1.820$ & 0.069 \\
\hline AT III(\%) & 950 & $60.69 \pm 17.87$ & $\begin{array}{l}68.39 \pm \\
18.54\end{array}$ & $t=-4.892$ & 0.0001 \\
\hline \multicolumn{6}{|c|}{$\begin{array}{l}\text { Note: * WBC } \llbracket \text { white blood cell. } \mathrm{N} \text { : percentage of neutrophil. L: percentage of lymphocyte. M: } \\
\text { percentage of monocyte. hsCRP: high sensitive } \mathrm{C} \text { reactive protein. }\end{array}$} \\
\hline \multicolumn{6}{|c|}{$\begin{array}{l}\text { PCT: procalcitonin. BUN: blood urea nitrogen. Cr: creatinine. TBIL: total bilirubin. PLT: platelet. AT III: } \\
\text { antithrombin III. }\end{array}$} \\
\hline \multicolumn{6}{|c|}{$\star \star X^{2}$ : Chi-square. t: t-test. Z: Mann Whitney test. } \\
\hline
\end{tabular}

\section{Clinical Intervention And Grouping:}

Patients were treated according to the clinical situation, including microculture, anti-infective therapy, fluid resuscitation, mechanical ventilation, hemo-purification and so on, and blood samples were drawn as needed. Monitoring the blood routine, liver and kidney function, blood coagulation function and other indicators of the patient is determined by the doctor to inject the polyunsaturated fatty acid into the patient, and the grouped according to whether the polyunsaturated fatty acid is used. polyunsaturated fatty acid(Fish oil fatty acid preparations) dosage: Intravenous injection $10 \mathrm{~g} /$ day, used with other fatty acid preparation at a 1:5 ratio. The patient's treatment is at the discretion of the doctor. In this retrospective study, no intervention was conducted on the initiation and cessation of treatment regimens.

Observation Indicators

Observation indicators were as follows: gender, age, respiration, heart rate, blood pressure, blood purification, mechanical ventilation, vasoactive drugs, blood routine, hypersensitive C-reactive protein (hsCRP), procalcitonin (PCT), liver and kidney function, clotting function (AT III, APTT)

statistic analysisSPSS 20.0 and R software package were used to analyze the data. Normally distributed continuous data were expressed as mean \pm standard deviation(SD), non-normal distribution values were presented as medians. The count values between the two groups are calculated by independent-sample $t-$ 
test, or Mann Whitney test, and the 95\% confidence interval(confidence index, $\mathrm{Cl}$ ) was calculated. Multivariate logistic regression analysis to evaluate the prognostic effect of other factors on the application of polyunsaturated fatty acids in the treatment of sepsis. Kaplan-Meier (KM) survival curves were plotted. The relationship between the total amount of polyunsaturated fatty acid use, ventilator use time, and ICU hospitalization days was evaluated by fitting curve and bubble diagram respectivel. $p<0.05$ denoted statistical significance.

\section{Results}

\section{Baseline characteristics}

Between December 2016 to June 2019, a total of 1,997 patients were collected. After applying inclusion and exclusion criteria, tha final sample consisted of 1733 patients. The patient's age, gender, site of infection, laboratory test indicators, etc. are shown in Table-1. Since the APACHE and SOFA scores of these patients cannot be obtained in the retrospective study, the evaluation of the main organ indicators were shown in table-2.

\section{Motality comparison}

Among the 303 cases in the polyunsaturated fatty acid treatment group, 96 cases died, with a case fatality rate of $31.68 \%$. The control group had 1,430 cases and 286 cases died. The case fatality rate was only $20.00 \%$. The case fatality rate of the treatment group was $11.68 \%$ higher than that of the control group(Fig. 1A, p囚0.0001). Most patients useed omega-3 fatty acids in the early stage(Fig. 1B). However, we can see from Table-2 that there are big differences between the two groups of patients. Age, gender, whether there is abdominal infection, whether sepsis, shock, the need for mechanical ventilation, and the need for renal replacement therapy may all affect the prognosis of the patient. Multivariate logistic regression analysis was conducted by considering death as end point, polyunsaturated fatty acids as factor, and age, sex, abdominal infection, sepsis, shock, mechanical ventilation, and renal replacement therapy as covariables, the result indicated there was no significant difference in mortality between the treatment group and the control group $(p=0.574$, Table 3$)$. 
Table 3

Multiple logistic regression



According to the results of single factor analysis in Table-1, there are differences in gender, age, whether there is abdominal infection, sepsis, whether mechanical ventilation, shock, renal replacement therapy, etc. Then analyzed the interactive effects using logistic regression, the significant $P$ value of interactive effect is 0.574 (Table 3 ).

3. Influence of omega-3 fatty acids on sepsis outcome

Take the death of the patient as the clinical outcome, and the time of transferring or ending the intensive care medicine department as the observation end date, KM curve survival curves were plotted. At the 30day outcome, the survival of patients in the omega-3 fatty acid treatment group was lower than that in the control group $(p=0.007)$, while the 60 -day outcome indicated that the survival of the patients in the omega-3 fatty acid treatment group was slightly higher than that of the control group $(p=0.062)$.

Moreover, The survival rate of patients in the omega-3 fatty acid treatment group who ICU stay more than 60 days was higher than that in the control group ( $p=0.035$,Fig. 2$)$.

\section{Discussion}

Sepsis is a serious syndrome. There are an estimated 31.5 million sepsis and 19.4 million severe sepsis cases worldwide. There may be 5.3 million deaths each year, which has become a heavy burden on the world[7]. Sepsis is newly defined as fatal multiple organ failure (MODS) caused by severe infection, which weakens the role of systemic inflammatory response syndrome (SIRS) [8]. However Inflammation still plays an important role in the occurrence and development of sepsis. The MODS and sepsis-induced death are mainly due to the strong inflammatory response in the initial stage of severe infection and the 
imbalance of the anti-inflammatory response in the late stage[9]. Thus it can be seen that in sepsis inflammation cannot be ignored, anti-inflammation is still an important aspect of sepsis treatment. omega-3 fatty acid, as an nutrition agent, the therapeutic effect of has been controversial. In this study, there are 303 patients accepted omega-3 fatty acid supplement at the early stage of sepsis. Although analysis suggests that patients receiving omega- 3 fatty acid treatment have a higher mortality rate than the control group, multi-factor analysis showed that these patients had higher disease severity than the control group. After removed these factors that affect the prognosis by multivariate logistic regression analysis, it was found that there was no difference in the mortality of the two groups of patients. This suggests that for relatively critically ill patients, doctors are more inclined to use omega- 3 fatty acids. Multivariate logistic regression analysis removed these factors that affect the prognosis and found that, in fact, there was no difference in the mortality of the two groups of patients. On the contrary, from the survival curve, as time goes by, patients treated with omega-3 fatty acids seem to have a better clinical prognosis. Judging from the improvement in the long-term prognosis of patients with sepsis after treatment with omega-3 fatty acids, it seems to consistent with the results of our previous randomized controlled trials[5].

Patients with sepsis often suffer from obvious hypoproteinemia due to reduced protein synthesis and increased consumption, which affects serum arachidonic acid (AA), eicosapentaenoic acid (EPA), and docosahexaene Metabolism of fatty acids such as DHA, and low AA levels are an important determinant of the prognosis of patients with sepsis[10]. In the study of Oz HS et al, rats were given diets of homocysteine, high omega-3 fatty acid group and non-omega-3 fatty acid group. After injection of endotoxin, these rats experienced different degrees of weight loss and tissue damage, and received omega-3 fatty acids. The liver enzymes and pathological indexes of diet rats are relatively better than other diet groups[11]. Treated mouse bone marrow-derived dendritic cells with n-3 fatty aciddocosahexaenoic acid (DHA), following stimulation with different toll-like receptor (TLR) ligands. Flow cytometry detects cell surface maturation markers and intracellular activity. Real-time RT-PCR and ELISA to detect the expression and secretion of cytokines. DHA maintains the immature phenotype of bone marrow-derived DC by preventing the up-regulation of MHCIl and costimulatory molecules (CD40, CD80, and CD86) and maintaining a high level of endocytic activity. DHA inhibits the production of proinflammatory cytokines by DCs stimulated by TLR2, 3, 4 and 9 ligands, including the interleukin IL-12 cytokine family (IL-12p70, IL-23 and IL-27). The inhibition of IL-12 expression by DHA is mediated by activating PPARY and inhibiting nuclear translocation of NFKAPABP65. Showed that DHA has antiinflammatory effects in the body[12].

In both the cecal ligation and puncture mouse model and patients with sepsis, it was found that dendritic cells were depleted, concluded that the process was related to increased apoptosis $\square$ The loss of DCs caused by this sepsis occurs after the activation of CD $3+C D 4+T$ cells and the loss of lymph nodes. Before the loss of DCs, there is no continuous increase in their mature state. Both mature and immature DCs are easily lost. CD8 + DCs are preferential loss of local and distant lymph nodes, this suggests the key role of DCs in sepsis[13]. Recent studies have discovered that in the early stages of inflammation, activated dendritic cells are characterized by reduced antigen cross-presentation capacity of newly 
discovered antigens and decreased production of immunogenic cytokines. The immunosuppression induced by sepsis is mainly due to the depletion of mature immature dendritic cells. On the other hand Immune tolerance in the late stage can cause DCs to release tumor necrosis factor inhibitory cytokines and participate in the maintenance of a local tolerance environment characterized by Treg cell aggregation[14]. By targeting DCs, it is found that the loss of the number and function of DCs caused by sepsis is one of the reasons for the deficiency of CD8 T cell immune function, and the treatment method to improve the status of DC cells after sepsis may contribute to the immune function of CD8 T cells recovery[15]. DHA pretreatment of DC can prevent LPS-induced DC maturation, maintain low expression of costimulatory molecules, and pro-inflammatory cytokines (IL-12p70, IL-6 and IL-23). T cells co-cultured with DC-DHA express higher levels of TGF $\beta$ and Foxp3, but do not show a functional Treg phenotype. Similar to the results of in vitro experiments, the beneficial effects of DHA are related to the decrease in the number of IFNY and IL-17 produced in the spleen and central nervous system, resulting in a decrease in the number of $\mathrm{CD} 4^{+} \mathrm{T}$ cells[16].In addition, omega-3 fatty acids also have an effect on other inflammatory cells. Studies have found that it can improve the ability of neutrophils to remove the pathogen, which mainly related to DHA [17]. EPA plays a certain role in inhibiting the migration of neutrophils to the lesion site. Studies have found that endothelial cells can participate in the migration of neutrophils by producing prostaglandin D2. When prostaglandin D2 binds to the receptor DP-1 on neutrophils, it causes neutrophils to adhere and migrate. Endothelial cells pretreated with EPA may reduce the production of prostaglandin D2 by endothelial cells and increase the production of prostate D3, thereby inhibiting the migration of neutrophils[18]. At the same time, for polyunsaturated fatty acids have multiple double bonds in their carbon chains, each double bond will cause the carbon chain to bend, so the accumulation of polyunsaturated fatty acids in the cell membrane cannot be as tight as saturated fatty acids. This increases the fluidity of immune inflammatory cells, reduces fragility, and plays an important role in prolonging cell life[19]. This effect can also appear in other different types of immune cells, and it can adjust the patient's immune mechanism. Since the chemotaxis, migration, and pathogen clearance of leukocytes are mainly in the early stages of inflammatory response, omge-3 fatty acids may affect this cell behavior in the early stage.

In the long term, the effect of Omega-3 fatty acids on inflammatory cells may be to inhibit cell DNA methylation. Fatty acids can modify DNA methylation in vitro, and in vivo studies have found that total DNA methylation and gene specific DNA methylation of PDK4 are positively correlated with eicosapentaenoic acid and arachidonic acid. Postprandial HDAC4 methylation is negatively correlated with arachidonic acid[20]. A study found that 174 Alzheimer's disease (AD) patients received 1.7g DHA and $0.6 \mathrm{~g}$ EPA or placebo every day for 6 months. It was found that patients receiving omega-3 fatty acid treatment had peripheral blood white blood cells (PBLs). ) 2 out of $4 \mathrm{CpG}$ sites have significantly reduced methylation. Hypomethylation of $\mathrm{CpG} 2$ and $\mathrm{CpG} 4$ sites is negatively correlated with changes in plasma EPA concentration, but no related to the changes of plasma DHA concentration[21]. However, it is still unclear whether omega-3 fatty acids supplemented parenterally can also demethylate blood cell DNA. However, bioinformatics studies have found that the methylation of IL- 6 promoter cg01770232 is related to the increase of IL- 6 concentration, and higher concentrations of omega- 3 fatty acids inhibit the 
methylation of IL6 promoter cg01770232, which can also inhibit IL. -6 expression, the relationship between n-3 polyunsaturated fatty acids and cg01770232 methylation depends on the rs2961298 genotype[22]. IL-6 is an early indicator of inflammatory response. For severe inflammatory response caused by severe infection, patients can soar within 24 hours and then rapidly decrease[23]. It is a sensitive and specific indicator for early diagnosis of sepsis. Therefore, the increased blood concentration of omega-3 fatty acids can inhibit the DNA methylation process and have an inhibitory effect on early inflammation.

Although omga-3 fatty acids may improve the prognosis of patients with sepsis, the dosage and method of use are not yet clear. In our department of intensive care medicine, a formula of $n 3: n 6=1: 5$ is generally used, and the total dose of omega- 3 fatty acids used by each patient is different. This study was the first attempt to analysis the information system in the hospital, therefore, there was also a serious imbalance between the control group and the treatment group, which had a certain impact on the data analysis and results. And retrospective research, unable to intervene in the treatment plan, and existing data can not be used to score patients and evaluate side effects. Nonetheless, we tried to analyzed the patient's organ failure data, and evaluated the patient's condition from various organ functions and adjuvant treatments. Due to the different severity of the patient's condition, only statistical methods can be used to adjust the patient's data. Paired studies may help to obtain more precise results and conclusions.

\section{Conclusion}

For patients with more severe sepsis, doctors are more inclined to use omega-3 fatty acids in early stage. Omega-3 fatty acids may have a certain effect on improving the long-term prognosis of sepsis, but the conclusions still need to be carefully accepted. Because the clinical conditions of the patients are different, the results of the paired study may be able to draw more precise conclusions and results.

\section{Declarations}

\section{Availability of data and materials}

All analyzed data are included in this published article. The original data are available upon reasonable request to the corresponding author.

\section{Ethics approval and consent to participate}

Not applicable.

\section{Consent for publication}

Not applicable.

\section{Competing interests}


The authors declare that they have no competing interests.

\section{Funding}

Not applicable.

\section{Authors' contributions}

XYL designed the study. HSC wrote the draft. CYH collected the data ,HDZ analyzed the data. YLC performed the data analyses and wrote the manuscript.XYL approved the final manuscript. All authors read and approved the final manuscript.

Acknowledgements

We are grateful to Dr. Chenxi Li , Dr. Xi Wang , Dr. Zhenmi Liu for suppling us additional data. We also acknowledge the time that Dr.Zhiqiang Zhou and Dr.Shunkang Wen took to respond to our queries.

\section{References}

1. Skulas-Ray AC. Omega-3 fatty acids and inflammation: a perspective on the challenges of evaluating efficacy in clinical research. Prostaglandins Other Lipid Mediat.2015;116-117:104 - 11.

2. Calder PC. Omega-3 fatty acids and inflammatory processes: from molecules to man. Biochem Soc Trans. 2017;45(5):1105-15.

3. Mo YP, Hu XL, Chang LL, Ma P. The effect of w-3 fatty acid supplementation in parenteral nutrition on the outcome of patients with sepsis: a systematic review and meta-analysis. Zhonghua Wei Zhong Bing Ji Jiu Yi Xue. 2014;26(3):142-7.

4. Chen HS, Wang W, Hong YC, Zhang HD, Hong CY, Liu XY. Single-blinded, randomized, and controlled clinical trial evaluating the effects of Omega-3 fatty acids among septic patients with intestinal dysfunction: a plot study. Experimental Therapeutic Medicine. 2017;14(2):1505-11.

5. Chen HS, Wang W, Hong CY, Zhang M, Hong YC, Wang S, Zhang HD. Omega-3 fish oil reduces mortality due to severe sepsis with acute gastrointestinal injury grade III. Phcogn Mag. 2017;13:40712.

6. Chen HS, Wang S, Zhao Y, Luo YT, Tong HS, Su L. Correlation analysis of omega-3 fatty acids and mortality of sepsis and sepsis-induced ARDS in adults: data from previous randomized controlled trials. Nutr J. 2018;17(1):57.

7. Fleischmann C, Scherag A, Adhikari NK, Hartog CS, Tsaganos T, Schlattmann P, et al. Assessment of Global Incidence and Mortality of Hospital-treated Sepsis. Current Estimates and Limitations. Am J Respir Crit Care Med. 2016;193(3):259-72.

8. Levy MM, Evans LE, Rhodes A. The Surviving Sepsis Campaign Bundle: 2018 update. Intensive Care Med. 2018;44(6):925-8. 
9. Delano MJ, Ward PA. The immune system's role in sepsis progression, resolution, and long-term outcome. Immunol Rev. 2016;274(1):330-53.

10. Yamaguchi J, Kinoshita K, Ihara S, Furukawa M, Sakurai A. The Clinical Significance of Low Serum Arachidonic Acid in Sepsis Patients with Hypoalbuminemia. Intern Med. 2018;57(13):1833-40.

11. Oz HS, Chen TS, Neuman M. Nutrition intervention: a strategy against systemic inflammatory syndrome. JPEN J Parenter Enteral Nutr. 2009;33(4):380-9.

12. Kong W, Yen JH, Vassiliou E, Adhikary S, Toscano MG, Ganea D. Docosahexaenoic acid prevents dendritic cell maturation and in vitro and in vivo expression of the IL-12 cytokine family. Lipids Health Dis. 2010;9:12.

13. Efron PA, Martins A, Minnich D, Tinsley K, Ungaro R, Bahjat FR, et al. Characterization of the systemic loss of dendritic cells in murine lymph nodes during polymicrobial sepsis. J Immunol. 2004;173(5):3035-43.

14. Bouras M, Asehnoune K, Roquilly A. Contribution of Dendritic Cell Responses to Sepsis-Induced Immunosuppression and to Susceptibility to Secondary Pneumonia. Front Immunol. 2018;9:2590.

15. Strother RK, Danahy DB, Kotov DI, Kucaba TA, Zacharias ZR, Griffith TS, et al. Polymicrobial Sepsis Diminishes Dendritic Cell Numbers and Function Directly Contributing to Impaired Primary CD8 T Cell Responses In Vivo. J Immunol. 2016;197(11):4301-11.

16. Kong W, Yen JH, Ganea D. Docosahexaenoic acid prevents dendritic cell maturation, inhibits antigenspecific Th1/Th17 differentiation and suppresses experimental autoimmune encephalomyelitis. Brain Behav Immun. 2011;25(5):872-82.

17. Svahn SL, Ulleryd MA, Grahnemo L, Ståhlman M, Borén J, Nilsson S, et al. Dietary Omega-3 Fatty Acids Increase Survival and Decrease Bacterial Load in Mice Subjected to Staphylococcus aureusInduced Sepsis. Infect Immun. 2016;84(4):1205-13.

18. Tull SP, Yates CM, Maskrey BH, O'Donnell VB, Madden J, Grimble RF, et al. Omega-3 Fatty acids and inflammation: novel interactions reveal a new step in neutrophil recruitment.PLoS Biol. 2009; 7(8):e1000177.

19. Hashimoto M, Hossain S. Fatty Acids: From Membrane Ingredients to Signaling Molecules. In: Waisundara V, editor. Biochemistry and Health Benefits of Fatty Acids. London: IntechOpen Limited; 2018.

20. de la Rocha C, Pérez-Mojica JE, León SZ, Cervantes-Paz B, Tristán-Flores FE, Rodríguez-Ríos D, et al. Associations between whole peripheral blood fatty acids and DNA methylation in humans. Sci Rep. 2016;6:25867.

21. Karimi M, Vedin I, Freund Levi Y, Basun H, Faxén Irving G, Eriksdotter M, et al. DHA-rich n-3 fatty acid supplementation decreases DNA methylation in blood leukocytes: the OmegAD study. Am J Clin Nutr. 2017;106(4):1157-65.

22. Ma Y, Smith CE, Lai CQ, Irvin MR, Parnell LD, Lee YC, et al. The effects of omega-3 polyunsaturated fatty acids and genetic variants on methylation levels of the interleukin- 6 gene promoter. Mol Nutr Food Res. 2016;60(2):410-9. 
23. Qiu X, Zhang L, Tong Y, Qu Y, Wang H, Mu D. Interleukin-6 for early diagnosis of neonatal sepsis with premature rupture of the membranes: A meta-analysis. Medicine. 2018;97(47):e13146.

\section{Figures}

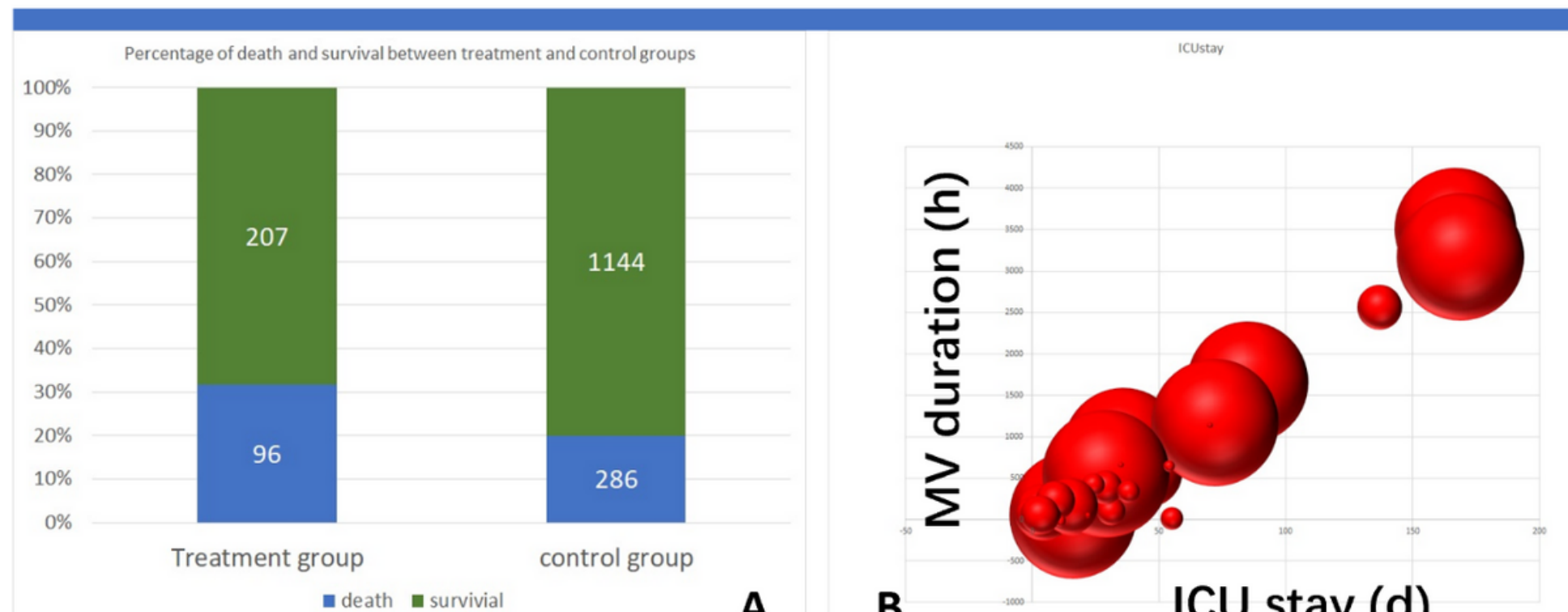

- death a survivial

A

B

ICU stay (d)

\section{Figure 1}

Omega-3 fatty acid on the prognosis of septic patients (Note $\mathbb{1} \mathrm{A} \rrbracket$ mortality of omega-3 fatty was higher than that of control group. 2B囚Bubble chart: the size of red bubble indicates the total dose of omega-3 fatty acid treatment. In most patients, omega-3 fatty acid was applicated in early state of treatment. $\mathbb{Z}$

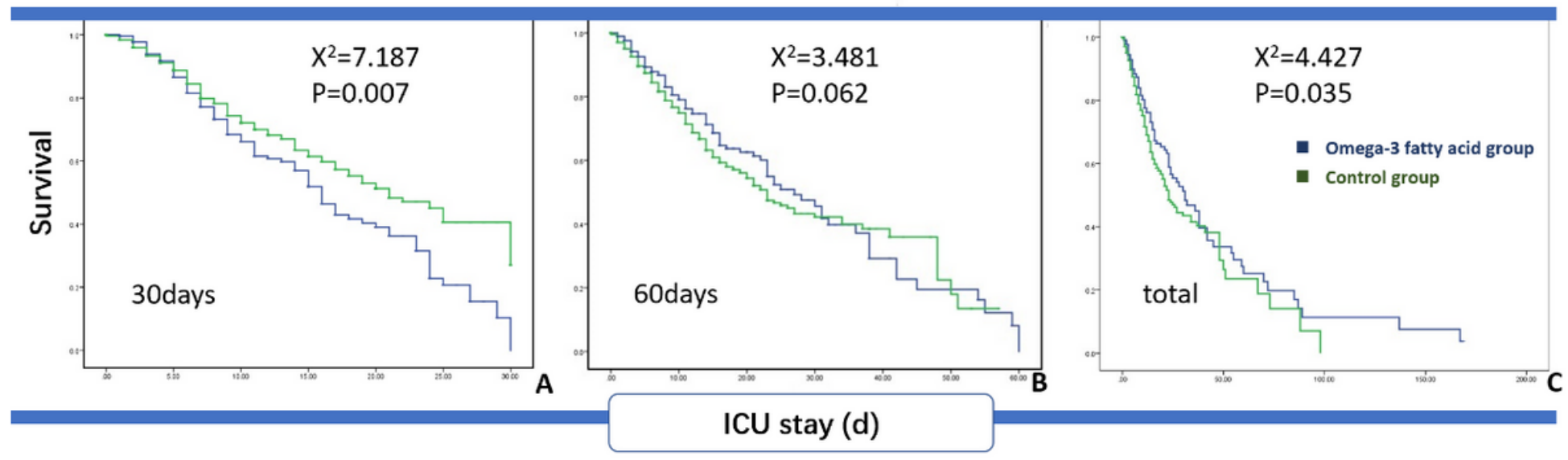

\section{Figure 2}

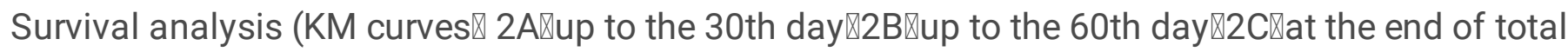

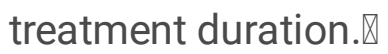

